\title{
Vascular flora in public spaces of Santiago, Chile
}

\section{Flora vascular en el espacio público de Santiago, Chile}

\author{
Javier A. Figueroa ${ }^{1 *}$, Sebastián Teillier ${ }^{2}$, Nicole Guerrero-Leiva ${ }^{3,4}$, Cristian Ray-Bobadilla ${ }^{3}$, \\ Simoné Rivano ${ }^{2}$, Diego SaAvedra ${ }^{3}$ \& Sergio A. Castro 3,4 \\ ${ }^{1}$ Centro de Estudios Arquitectónicos, Urbanísticos y del Paisaje, Facultad de Arquitectura, Urbanismo y Paisaje, Universidad \\ Central de Chile. Avenida Santa Isabel 1186, Santiago. \\ ${ }^{2}$ Escuela de Arquitectura del Paisaje, Universidad Central de Chile. Avenida Santa Isabel 1186, Santiago. \\ ${ }^{3}$ Laboratorio de Ecología y Biodiversidad Vegetal, Departamento de Biología, Universidad de Santiago de Chile. Casilla 40, \\ Correo 33, Santiago. Av. Lib. B. O’Higgins 3363, Santiago, Chile. \\ ${ }^{4}$ Centro para el Desarrollo de la Nanociencia y Nanotecnología, CEDENNA, 917-0124 \\ Santiago, Chile. Av. Lib. B. O’Higgins 3363, Santiago, Chile. \\ *javier.figueroa@ucentral.cl
}

\begin{abstract}
After an extensive two-year long research effort, the results document the diversity of vascular plants that grow in the public spaces of Santiago, Chile. We analyze the taxonomic composition, life-forms and phytogeographic origin of the vascular flora of Santiago and, finally, we compare the results with those of urban areas in the Northern Hemisphere. We identified 508 species, 100 families, and 338 genera. The families that showed the greatest richness were Asteraceae and Poaceae. We found that at least $85.1 \%$ of the species are exotic. The life-forms are similarly represented, although chamaephytes and geophytes are poorly represented. We conclude that the composition of the urban flora of Santiago differs from that of most Northern Hemisphere cities, due to the increased presence of exotic species, which is likely a consequence of the historical and cultural patterns of ornamentation. Therefore it is likely that this urban area would be an adverse environment for the establishment and development of native species.
\end{abstract}

KEYwords: Central Chile, exotic plants, native plants, plant diversity, urban flora.

\section{RESUMEN}

Los resultados documentan la diversidad de plantas vasculares que se desarrollan en el espacio público de Santiago en una investigación que se prolongó por dos años. Nosotros analizamos la composición taxonómica, la forma de vida y el origen fitogeográfico de la flora vascular y, finalmente, comparamos los resultados con aquellos de áreas urbanas del Hemisferio Norte. Se reconocen 508 especies, 100 familias y 338 géneros. Las familias que tienen mayor riqueza fueron Asteraceae y Poaceae. El 85,1\% de las especies son exóticas. Las formas de vida están similarmente representadas, aunque las caméfitas y las geófitas están muy poco representadas. Concluimos que la composición de la flora urbana de Santiago se distingue de la mayoría de las ciudades del Hemisferio Norte por el mayor número de especies exóticas, que es probablemente una consecuencia de los patrones históricos y culturales de ornamentación, al tiempo que los espacios urbanos parecen representar un ambiente adverso para el establecimiento y desarrollo de especies nativas.

Palabras Clave: Chile central, plantas exóticas, plantas nativas, biodiversidad de plantas, flora urbana.

\section{INTRODUCTION}

Cities are biodiversity centers that contain native and exotic plants in variable proportions (Kowarik et al. 2013, Pyšek 1998). Due to the multiple ecosystem services provided by urban biodiversity, and particularly, plants (i.e. decontamination, aesthetic, recreational, native biotic, and soil conservation), there has been a recent increase in studies on urban flora (Kowarik 2011, Gong et al. 2013, Kowarik et al. 2013).

Most studies analyzing the diversity of urban flora have been carried out in cities in the Northern Hemisphere, particularly in Europe and the United States (Burton 1983, Klotz 1990, Godefroid 2001, McKinney 2002, 2006, Chocholoušková \& Pysek 2003, Celesti-Grapow et al. 2006, Ricotta et al. 2009, Kowarik et al. 2013). These studies 
indicate that most of the flora in these cities are native species, representing approximately 60 to $40 \%$ of species present (Ricotta et al. 2009). Native richness within these cities has favored comprehensive conservation strategies (Sukopp \& Werner 1983, Pyšek 1998, Kühn et al. 2004, Celesti-Grapow et al. 2006, La Sorte \& McKinney 2006).

Studies on the flora and vegetation of urban areas in South America and, particularly in Chile, have been rather scarce (Cursach et al. 2012). However, there have been a few studies undertake in Chilean cities on such topics as: vegetational cover studies (Reyes-Paecke \& Meza 2011, De la Barrera et al. 2011), conservation of native flora and fauna species (Díaz \& Armesto 2003, Romero et al. 2001, Pauchard et al. 2006), the importance of social and economic factors in determining the value of vegetational cover (Romero et al. 2007, Hernández et al. 2007, Escobedo et al. 2008), the effects of pollen on human health (Rojas et al. 1999, Ibañez et al. 2001), and on the role of vegetational patterns in territorial planning (Romero et al. 2007). However, as far as we know, there are only two studies on the diversity of urban plants: Hoffmann (1998) and Alvarado et al. (2013). Thus, a central question remains: which vascular plant species grow in the capital city of Chile, and what is the proportion of native flora compared to the total number of species?

Based on evidence available in the published literature, this study hypothesized that exotic species are dominant in vascular flora in the public spaces of Santiago, Chile. After an extensive two-year long research effort, the results document the diversity of vascular plants that grow in Santiago's public spaces. Specifically, we analyzed the taxonomic composition, Raunkiaer's life-form, and the phytogeographic origin of the plant species and, finally, we compare the results with those of urban areas in the Northern Hemisphere.

\section{MATERIALS AND METHODS}

STUDY SITE

The city of Santiago, the capital of Chile $\left(33^{\circ} 27^{\prime} 00^{\prime \prime} \mathrm{S}\right.$ $70^{\circ} 40^{\prime} 00^{\prime \prime} \mathrm{W} ; 500$ masl), has a mediterranean-type climate. It was founded by the Spaniards in 1541. Today it covers an area of approximately $15,400 \mathrm{~km}^{2}$ and concentrates more than 6 million inhabitants (approximate density: 393 inhabitants $\left./ \mathrm{km}^{2}\right)^{1}$. Thus, it is one of the most densely concentrated urban areas in Latin America.

\section{SAMPLING}

To evaluate the floristic composition we established 200 random sites in public spaces of 35 communes within the city of Santiago (Fig. 1). These sites were georeferenced and visited between August and December in 2012 and again in 2013. At each site we placed a transect (100 $\mathrm{m}$ in

${ }^{1}$ URL: http://www.ine.cl/canales/chile_estadistico/familias/demograficas vitales.php Viewed: May 4, 2015. length by $1-3 \mathrm{~m}$ in width). For each species recognized we assigned the taxonomic category and phytogeographic origin according to Marticorena \& Quezada (1985), Matthei (1995), and Zuloaga et al. (2009). Several specimens were only determined at the genus level, thus they were not included in the analisys.

Species origins were classified as either native flora present in Chile prior to Spanish colonization or exotic flora that arrived afterwards. The categories presented by Raunkiaer (1934), as modified by Govaerts et al. (2000), were used to assign the life-form of each plant species: phanerophytes (have stems that are woody and persistent, and buds that are normally exposed $3 \mathrm{~m}$ or more above ground level), Nanophanerophytes (have stems that are woody and persistent, and buds typically located between $0.5 \mathrm{~m}$ and $3 \mathrm{~m}$ above ground level), chamaephytes (have stems that are herbaceous or woody and persistent, and have buds that are located above soil level, but never by more than $0.5 \mathrm{~m}$ ), hemicryptophytes (have herbaceous stems that often die-back during unfavourable seasons, and surviving buds placed on (or just below) soil level), terophytes (complete their entire life-cycle during the favourable season, and survive the unfavourable season as a seed), and geophytes (have stems that die back during unfavourable seasons, with the plant surviving as a bulb, rhizome, tuber or root bud).

The original distribution of the exotic flora collected in urban public space in Santiago, was determined consulting several sources (Tutin 1964-1983, Hoffmann 1998, Matthei 1995, Kuhn 2004). Thus, Euroasian origin includes Europa, near East and Mediterranean coast of Africa. American, African and Oceanian include original distribution from the respective continent. Asian origin includes species with distributional area on eastern Asia.

The collected specimens were deposited in the Laboratorio de Ecología y Biodiversidad Vegetal at the Universidad de Santiago de Chile.

\section{RESULTS}

At the 200 sites, we recognized 508 species, 100 families and 338 genera (Table I). Plants were determined at species level, and assigned to a taxonomic category as follows: Division Magnoliophyta (96.5\% of the species) and Pinophyta (3.5\% of the species). The four most diverse families within the urban flora of Santiago were as follows: Poaceae (54 species), Asteraceae (50 species), Fabaceae (36 species), and Rosaceae (31 species).

With respect to phytogeographic origin, 76 plant species were native to Chile (14.9\% of the identified origins), whereas that 432 were exotic $(85.1 \%$ of the identified origins). Within the exotic taxa the most common original distribution was Euroasian, representing $28.5 \%$ of the total (Table II), American (17.8\%), Asian (16.0\%), and African 


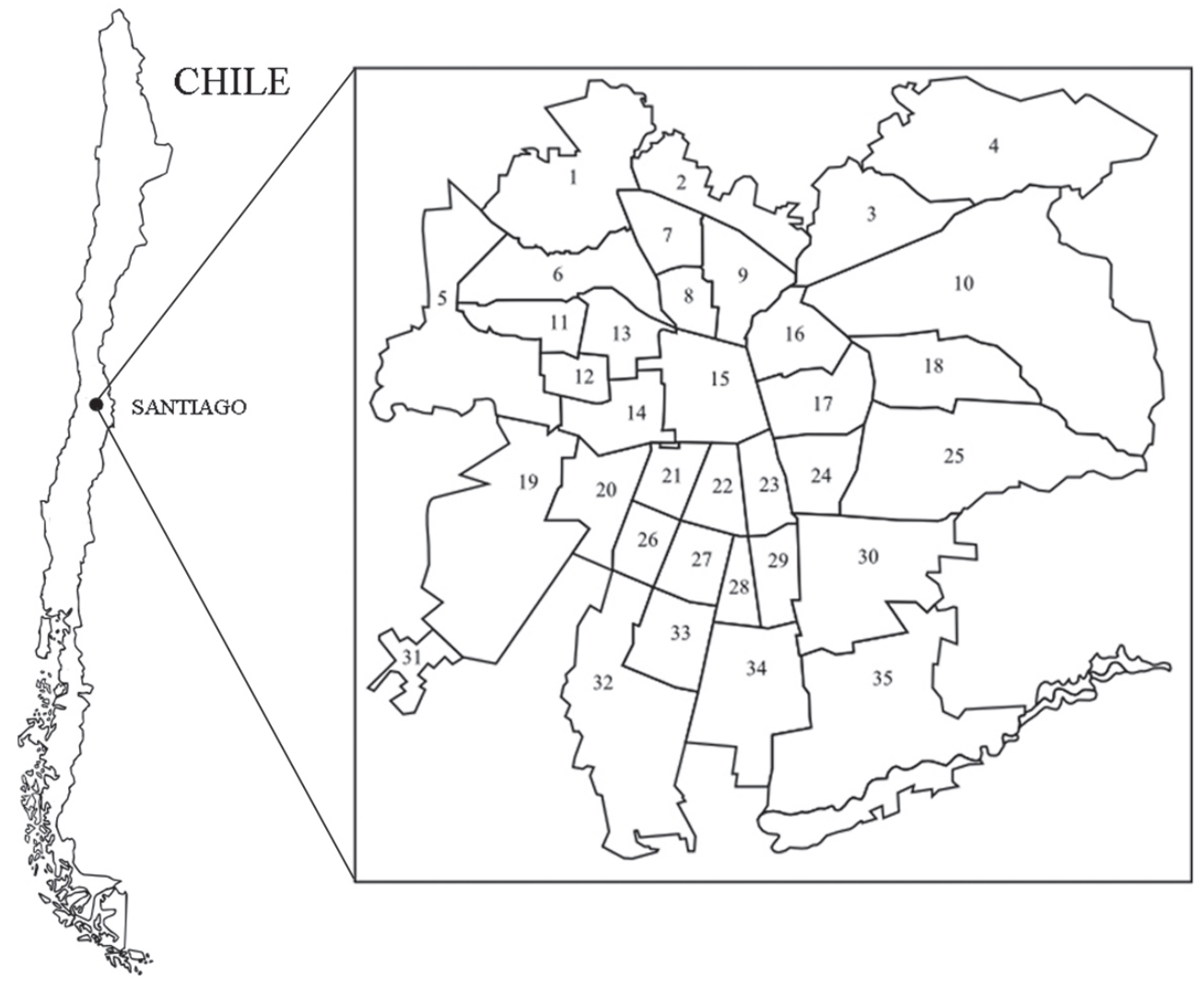

FIgURE 1. Location of the communes containing 200 study sites at Santiago, Chile. The communes of Santiago are showed. 1= Quilicura (4), 2= Huechuraba (3), 3= Vitacura (4), 4= Lo Barnechea (3), 5= Pudahuel (6), 6= Renca (6), 7= Conchalí (7), 8= Independencia (3), 9= Recoleta (7), 10= Las Condes (11), 11= Cerro Navia (4), 12= Lo Prado (4), 13= Quinta Normal (8), 14= Estación Central (7), 15= Santiago (9), 16= Providencia (8), 17= Ñuñoa (9), 18= La Reina (7), 19= Maipú (15), 20= Cerrillos (6), 21= Pedro Aguirre Cerda (5), 22= San Miguel (2), 23= San Joaquín (6), 24= Macul (4), 25= Peñalolén (8), 26= Lo Espejo (5), 27= La Cisterna (4), 28= San Ramón (3), 29= La Granja (5), 30= La Florida (7), 31= Padre Hurtado (1), 32= San Bernardo (4), 33= El Bosque (4), 34= La Pintana (5), 35= Puente Alto (6). Number of study sites sampled per commune are shown in parentheses.

Figura 1. Ubicación de las comunas que contienes los 200 sitios de estudio en Santiago de Chile. Las comunas de Santiago son señaladas. $1=$ Quilicura (4), 2= Huechuraba (3), 3= Vitacura (4), 4= Lo Barnechea (3), 5= Pudahuel (6), 6= Renca (6), 7= Conchalí (7), 8= Independencia (3), 9= Recoleta (7), 10= Las Condes (11), 11= Cerro Navia (4), 12= Lo Prado (4), 13= Quinta Normal (8), 14= Estación Central (7), 15= Santiago (9), 16= Providencia (8), 17= Nuñoa (9), 18= La Reina (7), 19= Maipú (15), 20= Cerrillos (6), 21= Pedro Aguirre Cerda (5), 22= San Miguel (2), 23= San Joaquín (6), 24= Macul (4), 25= Peñalolén (8), 26= Lo Espejo (5), 27= La Cisterna (4), 28= San Ramón (3), 29= La Granja (5), 30= La Florida (7), 31= Padre Hurtado (1), 32= San Bernardo (4), 33= El Bosque (4), 34= La Pintana (5), 35= Puente Alto (6). En paréntesis se señala el número de sitios de estudio muestreados por comuna.

TABLE I. Number of families, genera, and species identified ( $\mathrm{N}$ and \%) by taxonomic division of the urban flora in public space of Santiago, Chile.

TABLA I. Número de familias, géneros y especies identificadas ( $\mathrm{N}$ y \%) por división taxonómica de la flora urbana en el espacio público de Santiago, Chile.

\begin{tabular}{lcccc}
\hline Division & FAMILY & GenUS & SPECIES & $\%$ \\
\hline Magnoliophyta & 95 & 327 & 490 & 96.5 \\
Pinophyta & 5 & 11 & 18 & 3.5 \\
TOTAL & 100 & 338 & 508 & 100 \\
\hline
\end{tabular}


$(10.9 \%)$. Other distributional origins had representation $<10 \%$ (Table II); 11 exotic species were hybrids and considered with undetermined origin (Table II).

The most common life-forms was phanerophytes (137 species, Table III). Among these, 115 (83.9\%) were exotic and $22(16.1 \%)$ were native. Of particularly importance was the presence of endemic species such as: Aextoxicon punctatum Ruiz et Pav., Beilschmiedia berteroana (Gay) Kosterm., and B. miersii (Gay) Kosterm. The abundance of the following native phaneropytes was noteworthy: Quillaja saponaria Molina and Senna candolleana (Vogel) H.S. Irw. \& Barneby. We identified 131 terophytes, $87.0 \%$ of which were exotic species (Table III). It is interesting that among the 17 native terophytes, the most common were the following:

TABLE II. Original distribution of the exotic flora collected in public space in Santiago, Chile.

TABla II. Distribución de origen de la flora exótica colectada en espacios públicos de Santiago, Chile.

\begin{tabular}{lcc}
\hline ORIGINAL DISTRIBUTION & $\mathrm{N}$ & $\%$ \\
\hline Eurasian & 123 & 28.5 \\
American & 77 & 17.8 \\
Asian & 69 & 16.0 \\
African & 47 & 10.9 \\
Mediterranean & 42 & 9.7 \\
European & 32 & 7.4 \\
Oceanian & 18 & 4.2 \\
African and Asian & 8 & 1.9 \\
North American & 2 & 0.5 \\
American and African & 1 & 0.2 \\
Eurasian and North American & 1 & 0.2 \\
Oceanian and Asian & 1 & 0.2 \\
Undetermined origin (hybrids) & 11 & 2.5 \\
\hline TOTAL & 432 & 100 \\
\hline
\end{tabular}

TABLE III. Life form and origin of the exotic flora collected in public space in Santiago, Chile. Frequency (N) and percentage (\%) for each life form are indicaded.

TABLA III. Forma de vida y origen de toda la flora urbana colectada en espacios públicos de Santiago, Chile. Se señala la frecuencia (N) y el porcentaje (\%) para cada forma de vida.

\begin{tabular}{llcc}
\hline LIFE FORM & ORIGIN & N & $\%$ \\
\hline \multirow{2}{*}{ Phanerophytes } & Native & 22 & 16.1 \\
& Exotic & 115 & 83.9 \\
\hline \multirow{2}{*}{ Nanophanerophytes } & Native & 15 & 14.9 \\
& Exotic & 86 & 85.1 \\
\hline \multirow{2}{*}{ Hemicryptophytes } & Native & 17 & 15.9 \\
& Exotic & 90 & 84.1 \\
Terophytes & Native & 17 & 13.0 \\
& Exotic & 114 & 87.0 \\
\hline \multirow{2}{*}{ Chamaephytes } & Native & 4 & 16.0 \\
& Exotic & 21 & 84.0 \\
\hline \multirow{2}{*}{ Geophytes } & Native & 1 & 14.3 \\
& Exotic & 6 & 85.7 \\
\hline
\end{tabular}


Aristolochia chilensis Bridges ex Lindl., Loasa triloba Dombey ex Juss, and Oxalis rosea Jacq. Nanophanerophytes were represented by a sizable group of 101 species (Table III). Among these 86 species were exotic, and 15 native (e.g. Colliguaja odorifera Molina, Escallonia illinita C. Presl, Fuchsia magellanica Lam., and Luma chequen (Molina) A. Gray). Another important group consists of hemicryptophytes, among which 107 were exotic (84.1\%) and 17 were native, including Dysphania ambrosioides (L.) Mosyakin \& Clemants, Festuca acanthophylla E. Desv., and Pasithea caerulea (Ruiz. et Pav.) D. Don. We identified 21 exotic chamaephytes and 4 native chamaephytes, including Carpobrotus chilensis (Molina) N. E. Br., Fragaria chiloensis (L.) Mill., and Sphaeralcea obtusiloba G. Don. Finally, we identified 7 geophytes, of which 1 were native (Table III): Oxalis arenaria Bertero.

\section{DISCUSSION}

According to our results, the composition of the urban flora of Santiago differs from that of most Northern Hemisphere cities in terms of the greater representation of exotic species (Burton 1983, Klotz 1990, Godefroid 2001, McKinney 2002, 2006, Chocholouskova \& Pyšek 2003, Celesti-Grapow et al. 2006, Ricotta et al. 2009, Kowarik et al. 2013). The scarce representation of native species in Santiago suggests that this urban area may be an adverse environment for the establishment and development of their individuals. It is noteworthy that some native plants, such as Aextoxicon punctatum Ruiz. et Pav., Beilschmiedia spp. and Fuchsia magellanica Lam., were planted for ornamental or urbanistic purposes and there is no evidence that they grow spontaneously. In fact, during the colonial period of Chilean history and the $19^{\text {th }}$ century, Santiago was ornamented using exotic trees, following the model of European parks and gardens, and native species were scarcely used during $20^{\text {th }}$ and $21^{\text {st }}$ century (Serra et al. 2002, Hoffmann 1998, Alvarado et al. 2013).

This study shows that in Santiago's public spaces exotic plant species are more common than native species. In contrast, in diverse patches of vegetation in central Chile, the richness of exotic species is rarely greater than native ones (Teillier et al. 2010). The origins of the urban flora in Santiago are similar to that of plants found on the agricultural lands of central Chile, where exotic weeds represent about $80 \%$ of species (Figueroa et al. 2013). It is known that the successful establishment of exotic species is much more frequent in artificial habitats, and that they likely benefit from the high rate of anthropogenic disturbance and a largescale removal of the natural habitat (Sukopp 2004, Figueroa et al. 2013, Gong et al. 2013).

Given these results, and due to the present social and cultural conditions, the idea of considering Santiago as a spot for the conservation of native species should be undertaken soon. In many cities there are organizations willing to allocate resources to conservation and education efforts (Figueroa et al. 2011). Thus, the role of both private and public agencies needs to be coordinated in order to empower organizations within civil society.

Green spaces can be opportunities for ex-situ conservation and environmental education, while at the same time collaborating for efficient use of water and reducing the atmospheric pollen load (Rojas et al. 1999).

On the other hand, the predominance of Eurasian species seen in Santiago is consistent with the flora present in rural areas as well as with the overall flora of central Chile (Teillier et al.2010). This is most likely a consequence of the high historical availability of Eurasian plants in the country (Figueroa et al. 2004). It is worth noting that African and Oceanic species represented the $15.1 \%$ of the species pool in Santiago, far higher that the $3 \%$ of species they represent in natural habitats of central Chile (Teillier et al. 2010). Clearly, African and Oceanic species require to be monitored, since various weeds and invasive plants currently commons in central Chile were initially introduced in Santiago during $19^{\text {th }}$ century and later they spread spontaneously to rest of the country (Matthei 1995). Although our study constitutes a random sampling of diversity only in public spaces, studies that include private spaces are required because these form an important contribution to urban diversity that is usually neglected (Smith et al. 2006).

This is the first floristic study that we knowing the literature that considers the non-woody species in public space of Santiago. Although in Santiago the non-woody (mainly hemicryptophytes and therophytes) cover is lower than woody cover, this study demonstrates that species richness is similar in both. While a large proportion of therophytes have spontaneously established themselves in the diverse artificial habitats available in the city, phanerophytes, on the other hand were cultivated in the city and they need to be managed by municipal agencies. Nevertheless, the source of therophytes is diverse although we were not able to accurately determine, and their impact on urban activities within Santiago has not yet been evaluated.

Nowadays there is an agreement that cities are not internally homogeneous structures, they are typically characterized by a wide ranging spatial and temporal heterogeneity caused by cultural, socio - economic and environmental patterns particular to each town (Gilbert 1989, Kowarik 1995, Kinzig \& Grove 2001, Pickett et al. 2001, Barbosa et al. 2007, Sánchez \& Bonilla 2007, Marco et al. 2010). There is a growing need of more research in order to determine the causes that are responsible for the current patterns of diversity in species composition and abundance of the urban flora of Santiago. 


\section{ACKNOWLEDGEMENTS}

This study was financed by the "Fondo Interno de Investigación, Universidad Central de Chile", and "Programa Regular de Investigación CEAUP”. S.A.C. thank financial support of Center for the Development of Nanoscience and Nanotechnology CEDENNA FB0807 (Línea 6), DICYT 021543CM and Proyecto USA1498.04 of the Universidad de Santiago de Chile.

\section{REFERENCES}

Alvarado, A., A. Bandini \& F. Guajardo. 2013. Árboles urbanos de Chile. Guía de reconocimiento. $2^{\mathrm{a}}$ edición. Corporación Nacional Forestal. Santiago. 376 pp.

Barbosa, O., J. Tratalos, P. Armsworth, R. Davies, R. Fueller, J. PAт \& K. Gaston. 2007. Who benefits with access from green space? A case study from Sheffield UK. Landscape and Urban Planning 83: 187-195.

Burton, R.M. 1983. Flora of the London area. London Natural History Society. Colchester. 225 pp.

Celesti-Grapow, L., P. Pyšek, V. Jarosík \& C. Blasi. 2006. Determinants of native and alien species richness in the urban flora of Rome. Diversity and Distributions 12: 490501.

Chocholouskova, Z. \& P. Pyšek. 2003. Changes in composition and structure of urban flora over 120 years: a case study of the city of Plzeo. Flora 198: 366-376.

Cursach, J.A., J.R. Rau, C. N. Tobar \& J.A. OJeda. 2012. Estado actual del desarrollo de la ecología urbana en grandes ciudades del sur de Chile. Revista de Geografía, Norte Grande 52: 57-70.

De la Barrera, F., G. Sepúlveda \& A. Oporto. 2011. Flora vascular asociada al sistema de esteros urbanos de Placilla de Peñuelas (Región de Valparaíso, Chile). Chloris Chilensis 14. No 1. URL: http://www.chlorischile.cl Viewed: May 4, 2015.

DíAz, I.A. \& J.J. Armesto. 2003. La conservación de las aves silvestres en ambientes urbanos de Santiago. Revista Ambiente y Desarrollo CIPMA (Chile) 29: 31-38.

Escobedo, F.J., J.E. Wagner, D.J. NowaK, C.L. DE la Maza, M. Rodríguez \& D.E. Crane. 2008. Analyzing the cost effectiveness of Santiago, Chile's policy of using urban forests to improve air quality. Journal of Environmental Management 86: 148-157.

Figueroa, J.A., S.A. Castro, P.A. Marquet \& F.M. Jaksic. 2004. Exotic plant invasions to the Mediterranean region of Chile: causes, history and impacts. Revista Chilena de Historia Natural 77: 465-483.

Figueroa, J.A., S. Teillier, G. Carvallo, S.A. Castro. 2013. Especies de plantas exóticas en los campos agrícolas y en los sitios perturbados en Chile Central. En: O. Reveco (ed.), Más allá de lo dicho: hallazgos desde la investigación II, pp. 369-384. Universidad Central de Chile, Santiago, Chile.

Figueroa, R., C. Chaparro, M. González \& J.A. Figueroa. 2011. Reviewing nacional experiences, Chile. In: I Mulà \& D. Tilbury (eds.), National Journeys towards Education for
Sustainable Development, pp. 17-35. UNESCO, Paris, France.

Gilbert, O.L. 1989. The ecology of urban habitats. Chapman and Hall. London. 369 pp.

Godefroid, S. 2001. Temporal analysis of the Brussels flora as indicator for changing environmental quality. Landscape Urban Plannig 52: 203-224.

Gong, C., J. Chen \& Y. Shixiao. 2013. Biotic homogenization and differentiation of the flora in artificial and near-natural habitats across urban green spaces. Lanscape and Urban Planning 120: 158-169.

Govaerts, R., D.G. Frodin \& A. Radcliffe-Smith. 2000. World checklist and bibliography of Euphorbiaceae (with Pandanaceae). The Royal Botanic Gardens. Kew. 1661 pp.

Hernández, J., M.T. Serra \& J. Araya. 2007. Manejo de vegetación urbana. En: J. Hernández, C.L. de la Maza \& C. Estades (eds.), Biodiversidad: Manejo y conservación de recursos forestales, pp. 693-719. Editorial Universitaria, Santiago, Chile.

Hoffmann, A. 1998. El árbol urbano en Chile. $3^{\text {a }}$ edición. Fundación Claudio Gay, Santiago. 79 pp.

IbaÑEz, V., G. RoJas \& J. Roure. 2001. Airborne fungi monitoring in Santiago, Chile. Aerobiologia 17: 137-142.

Kinzig, A.P. \& J.M. Grove. 2001. Urban-suburban ecology. In: S.A. Levin (ed.), Encyclopaedia of Biodiversity Vol. 5, pp. 733-745. Academic Press, San Diego, USA.

KLotz, S. 1990. Species/area and species/inhabitants relations in European cities. In: H. Sukopp, S. Hejnyy \& I. Kowarik (eds.), Urban ecology, pp. 99-103. SPB Academic Publishing, The Hague, Netherlands.

KowariK, I. 1995. On the role of alien species in urban flora and vegetation. In: P. Pysek, K. Prach, M. Rejmánek \& P.M. Wade (eds.), Plant Invasions: General Aspects and Special Problems, pp. 85-103. SPB Academic, Amsterdam, Netherlands.

KowarIK, I. 2011. Novel urban ecosystems, biodiversity and conservation. Environmental Pollution 159: 1974-1983.

Kowarik, I., M. von der Lippe \& A. CierJacks. 2013. Prevalence of alien versus native species of woody plants in Berlin differs between habitat and at different scales. Preslia 85: 113-132.

KüHn, I., R. BRANDL \& S. KLOtZ. 2004. The flora of German cities is naturally species rich. Evolutionary Ecology Research 6: 749-764.

La Sorte, F.A., Mckinney, M.L. 2006. Compositional similarity and the distribution of geographical range size for assemblages of native and non-native species in urban floras. Diversity and Distributions 12: 679-686.

Marco, A., C. Barthelemy, T. Dutoit \& V.B. Taudiere-Montes. 2010. Bridging human and natural sciences for a better understanding of urban floral patterns: The role of planting practices in Mediterranean gardens. Ecology and Society 15: 2-21.

Marticorena, C. \& M. Quezada. 1985. Catálogo de la flora vascular de Chile. Gayana Botánica 42: 1-157.

Matthei, O. 1995. Manual de malezas que crecen en Chile. Alfabeta Impresores. Santiago. 547 pp.

MCKInNEY, M.L. 2002. Urbanization, biodiversity, and conservation. BioScience 52: 883-890.

MCKINNEY, M.L. 2006. Urbanization as a major cause of biotic 
homogenization. Biological Conservation 127: 247-260.

Pauchard, A., M. Aguayo, E. Peña \& R. Urrutia. 2006. Multiple effects of urbanization on the biodiversity of developing countries: The case of a fast-growing metropolitan area (Concepción, Chile). Biological Conservation 127: 272281.

Pickett, S.T., M.L. Cadenasso, J.M. Grove, C.H. Nilon, R.V. Pouyat, W.C. Zipperer \& R. Constanza. 2001. Urban ecological system: Linking terrestrial ecological, physical, and socioeconomic components of metropolitan areas. Annual Review of Ecology and Systematics 32: 127-157.

PYŠEK, P. 1998. Alien and native species in central European urban floras: A quantitative comparison. Journal of Biogeography 25: $155-163$.

RAUNKIAER, C.C. 1934. The life forms of plants and statistical plant geography. Oxford University Press. Oxford. 632 pp.

Reyes-Paecke, S. \& I. Meza. 2011. Jardines residenciales en Santiago de Chile: Extensión, distribución y cobertura vegetal. Revista Chilena de Historia Natural 84: 581-592.

Ricotta, C., S. Godefroid \& D. Rocchini. 2009. Patterns of native and exotic species richness in the urban flora of Brussels: rejecting the 'rich get richer' model. Biological Invasions 12: $233-240$.

Rojas, G., J. Roure, F. Galleguillos, P. Mardones. 1999. Aeropalinología de Santiago. Revista Chilena de Enfermedades Respiratorias 15: 141-155.

Romero, H., M. Molina, C. Moscoso, P. Sarricolea, P. Smith \& A. VÁsQuez. 2007. Caracterización de los cambios de usos y coberturas de suelo causados por la expansión urbana de Santiago, análisis de sus factores explicativos e inferencias ambientales. En: C. de Mattos \& R. Hidalgo (eds.), Santiago de Chile: Movilidad espacial y Reconfiguración Metropolitana, pp. 251-269. Pontificia Universidad Católica de Chile, Santiago, Chile.

Romero, H., X. Toledo, F. Órdenes \& A. VÁsquez. 2001. Ecología urbana y gestión sustentable de las ciudades intermedias chilenas. Ambiente y Desarrollo 17: 45-51.

SÁnchez, R. \& A. Bonilla. 2007. Urbanization, global environmental change, and sustainable development in Latin America. São José dos Campos. 198 pp.

Serra, M.T., J. Torres \& I. Grez. 2002. Breve historia de la introducción en Chile del álamo (Populus nigra L. var. italica (Moench.) Koehne) y el desarrollo de ejemplares siempreverdes. Chloris Chilensis, Año 5, No 2 . URL: http://www.chlorischile.cl

Smith, R.M., K. Thompson, J.G. Hodgson, P.H. Warren \& K.J. GASTON. 2006. Urban domestic gardens (IX): composition and richness of the vascular plant flora, and the implications for native biodiversity. Biological Conservation 129: 312322.

Stevens, P.F. (2001 onwards). Angiosperm Phylogeny Website. Version 9, June 2008. URL: http://www.mobot.org/ MOBOT/research/APweb/Viewed: May 4, 2015.

SuKopp, H. 2004. Human-caused impact on preserved vegetation. Landscape and Urban Planning 68: 347-355.

SuKopp, H. \& P. WerNER. 1983. Urban environment and vegetation. In: W. Holzner, M.J.A. Werger \& I. Ikusima (eds.), Man's impact on vegetation, pp. 247-260. W. Junk Publishers, The Hague, Netherlands.

Teillier, S., J.A. Figueroa \& S.A. Castro. 2010. Especies exóticas de la vertiente occidental de la cordillera de la Costa, Provincia de Valparaíso, Chile central. Gayana Botánica 67: 27-43.

Tutin, T.G., V.H. Heywood, N.A. Burges, D.M. Moore, D.H. Valentine, S.M. Walters \& D.A. WebB. 1964-1983. Flora Europaea. Royal Botanic Garden Edinburgh, Edinburgh, Scotland.

Zuloaga, F., O. Morrone \& M. Belgrano. 2009. Catálogo de las plantas vasculares del Cono Sur. Versión base de datos en sitio web del Instituto Darwinion, Argentina. URL: http:// www.darwin.edu.ar/Proyectos/FloraArgentina/FA.asp Viewed: May 4, 2015.

ANNEX 1. Urban flora collected in the public space of Santiago, Chile ${ }^{1}$.

ANEXo 1. Flora urbana recolectada en los espacios públicos de Santiago, Chile

\begin{tabular}{lcccc}
\hline SPECIES & FAMILY & LifE FORM & NATIVE DISTRIBUTION & ORIGIN STATUS \\
\hline Acanthus mollis L. & Acanthaceae & Hemicryptophyte & Mediterranean & Exotic \\
\hline Sambucus nigra L. & Adoxaceae & Nanophanerophyte & Eurasian & Exotic \\
\hline Viburnum rotundifolium Raf. & Adoxaceae & Nanophanerophyte & Asian & Exotic \\
\hline Viburnum tinus L. & Adoxaceae & Nanophanerophyte & Mediterranean \\
\hline Viburnum lucidum Mill. & Adoxaceae & Nanophanerophyte & Mediterranean & Exotic \\
\hline Aextoxicon punctatum Ruiz \& Pav. & Aextoxicaceae & Phanerophyte & South American & Native \\
\hline Mesembryanthemum cordifolium L.f. & Aizoaceae & Hemicryptophyte & African & Exotic \\
\hline Carpobrotus chilensis (Molina) N.E. Br. & Aizoaceae & Chamaephyte & South American & Native \\
\hline Drosanthemum hispidum (L.) Schwantes & Aizoaceae & Chamaephyte & African & Exotic \\
\hline Liquidambar styraciflua L. & Altingiaceae & Phanerophyte & American & Exotic \\
\hline Amaranthus deflexus L. & Amaranthaceae & Chamaephyte & American & Exotic \\
\hline Beta vulgaris L. & Amaranthaceae & Terophyte & Mediterranean & Exotic \\
\hline Chenopodium album L. & Amaranthaceae & Terophyte & Eurasian & Exotic \\
\hline
\end{tabular}


Gayana Bot. 73(1), 2016

\begin{tabular}{|c|c|c|c|c|}
\hline SpecIES & FAMILY & LIFE FORM & Native distribution & ORIGIN STATUS \\
\hline Chenopodium hircinum Schrad. & Amaranthaceae & Terophyte & American & Exotic \\
\hline Chenopodium murale L. & Amaranthaceae & Terophyte & Eurasian & Exotic \\
\hline Chenopodium vulvaria $\mathrm{L}$. & Amaranthaceae & Terophyte & Eurasian & Exotic \\
\hline $\begin{array}{l}\text { Dysphania ambrosioides (L.) Mosyakin } \\
\text { \& Clemants }\end{array}$ & Amaranthaceae & Hemicryptophyte & South American & Native \\
\hline Agapanthus africanus (L.) Hoffmanns. & Amaryllidaceae & Geophyte & African & Exotic \\
\hline Allium neapolitanum Cirillo & Amaryllidaceae & Geophyte & Eurasian & Exotic \\
\hline Nothoscordum bivalve (Sims) P. Beauv. & Amaryllidaceae & Geophyte & American & Exotic \\
\hline Nothoscordum gracile (Aiton) Stearn & Amaryllidaceae & Geophyte & African & Exotic \\
\hline Lithrea caustica (Molina) Hook. \& Arn. & Anacardiaceae & Phanerophyte & South American & Native \\
\hline Rhus crenata Thunb. & Anacardiaceae & Nanophanerophyte & African & Exotic \\
\hline $\begin{array}{l}\text { Schinus latifolius (Gillies ex Lindl.) } \\
\text { Engl. }\end{array}$ & Anacardiaceae & Phanerophyte & South American & Native \\
\hline Schinus molle L. & Anacardiaceae & Phanerophyte & South American & Native \\
\hline Schinus polygama (Cav.) Cabrera & Anacardiaceae & Phanerophyte & South American & Native \\
\hline Ammi visnaga (L.) Lam. & Apiaceae & Terophyte & Mediterranean & Exotic \\
\hline Anthriscus caucalis M.Bieb. & Apiaceae & Terophyte & Mediterranean & Exotic \\
\hline Bowlesia incana Ruiz \& Pav. & Apiaceae & Terophyte & South American & Native \\
\hline Conium maculatum L. & Apiaceae & Terophyte & Eurasian & Exotic \\
\hline Coriandrum sativum L. & Apiaceae & Terophyte & Eurasian & Exotic \\
\hline $\begin{array}{l}\text { Cyclospermum leptophyllum (Pers.) } \\
\text { Sprague }\end{array}$ & Apiaceae & Terophyte & South American & Native \\
\hline Daucus carota $\mathrm{L}$. & Apiaceae & Terophyte & Asian & Exotic \\
\hline $\begin{array}{l}\text { Eryngium paniculatum Cav. \& Dombey } \\
\text { ex. F.Delaroche }\end{array}$ & Apiaceae & Chamaephyte & South American & Native \\
\hline Foeniculum vulgare Mill. & Apiaceae & Hemicryptophyte & Eurasian & Exotic \\
\hline Nerium oleander L. & Apocynaceae & Nanophanerophyte & Eurasian & Exotic \\
\hline $\begin{array}{l}\text { Thrachelospermum jasminoides (Lindl.) } \\
\text { Lem. }\end{array}$ & Apocynaceae & Nanophanerophyte & Asian & Exotic \\
\hline Vinca major $\mathrm{L}$. & Apocynaceae & Hemicryptophyte & Mediterranean & Exotic \\
\hline Vinca minor $\mathrm{L}$. & Apocynaceae & Hemicryptophyte & European & Exotic \\
\hline Monstera deliciosa Liebm. & Araceae & Hemicryptophyte & American & Exotic \\
\hline $\begin{array}{l}\text { Philodendron bipinnatifidum Schott ex } \\
\text { Endl. }\end{array}$ & Araceae & Nanophanerophyte & American & Exotic \\
\hline Syngonium podophyllum Schott & Araceae & Chamaephyte & American & Exotic \\
\hline Zantedeschia aethiopica (L.) Spreng. & Araceae & Hemicryptophyte & African & Exotic \\
\hline$\times$ Fatshedera lizei (Cochet) Guillaumin & Araliaceae & Nanophanerophyte & Artificial hybrid & Exotic \\
\hline $\begin{array}{l}\text { Fatsia japonica (Thunb.) Decne. \& } \\
\text { Planch. }\end{array}$ & Araliaceae & Nanophanerophyte & Asian & Exotic \\
\hline Hedera canariensis Willd. & Araliaceae & Nanophanerophyte & African & Exotic \\
\hline Hedera colchica (K.Koch) K.Koch & Araliaceae & Nanophanerophyte & Eurasian & Exotic \\
\hline Hedera helix $\mathrm{L}$. & Araliaceae & Nanophanerophyte & Eurasian & Exotic \\
\hline Schefflera arboricola (Hayata) Merr. & Araliaceae & Nanophanerophyte & Asian & Exotic \\
\hline Araucaria angustifolia (Bertol.) Kuntze & Araucariaceae & Phanerophyte & American & Exotic \\
\hline Araucaria araucana (Molina) K. Koch & Araucariaceae & Phanerophyte & South American & Native \\
\hline Araucaria bidwillii Hook. & Araucariaceae & Phanerophyte & Oceanian & Exotic \\
\hline Araucaria heterophylla (Salisb.) Franco & Araucariaceae & Phanerophyte & Oceanian & Exotic \\
\hline
\end{tabular}


Urban flora of Santiago: FigueroA, J.A. ET AL.

\begin{tabular}{|c|c|c|c|c|}
\hline SpeCIES & FAMILY & LIFE FORM & Native distribution & ORIGIN STATUS \\
\hline Phoenix canariensis Chabaud & Arecaceae & Phanerophyte & African & Exotic \\
\hline $\begin{array}{l}\text { Syagrus romanzoffiana (Cham.) } \\
\text { Glassman }\end{array}$ & Arecaceae & Phanerophyte & American & Exotic \\
\hline Trachycarpus fortunei (Hook.) H.Wendl. & Arecaceae & Phanerophyte & Asian & Exotic \\
\hline $\begin{array}{l}\text { Washingtonia filifera (Linden ex André) } \\
\text { H.Wendl. ex de Bary }\end{array}$ & Arecaceae & Phanerophyte & American & Exotic \\
\hline Aristolochia chilensis Bridges ex Lindl. & Aristolochiaceae & Terophyte & South American & Native \\
\hline Agave americana $\mathrm{L}$. & Asparagaceae & Hemicryptophyte & American & Exotic \\
\hline Asparagus densiflorus (Kunth) Jessop & Asparagaceae & Hemicryptophyte & African & Exotic \\
\hline Asparagus setaceus (Kunth) Jessop & Asparagaceae & Hemicryptophyte & African & Exotic \\
\hline $\begin{array}{l}\text { Chlorophytum comosum (Thunb.) } \\
\text { Jacques }\end{array}$ & Asparagaceae & Chamaephyte & African & Exotic \\
\hline Cordyline australis (G.Forst.) Endl. & Asparagaceae & Phanerophyte & Oceanian & Exotic \\
\hline Ruscus aculeatus L. & Asparagaceae & Hemicryptophyte & Mediterranean & Exotic \\
\hline Ruscus hypoglossum L. & Asparagaceae & Hemicryptophyte & Eurasian & Exotic \\
\hline Achillea millefolium $\mathrm{L}$. & Asteraceae & Hemicryptophyte & European & Exotic \\
\hline Anthemis cotula $\mathrm{L}$. & Asteraceae & Terophyte & Mediterranean & Exotic \\
\hline Arctotheca calendula (L.) Levyns & Asteraceae & Hemicryptophyte & African & Exotic \\
\hline $\begin{array}{l}\text { Argyranthemum frutescens (L.) Sch. } \\
\text { Bip. }\end{array}$ & Asteraceae & Nanophanerophyte & African & Exotic \\
\hline Baccharis linearis (Ruiz \& Pav.) Pers. & Asteraceae & Nanophanerophyte & South American & Native \\
\hline Bellis perennis L. & Asteraceae & Hemicryptophyte & Eurasian & Exotic \\
\hline Bidens aurea (Aiton) Sherff & Asteraceae & Hemicryptophyte & American & Exotic \\
\hline Calendula officinalis $\mathrm{L}$. & Asteraceae & Terophyte & Mediterranean & Exotic \\
\hline Callistephus $\times$ sinensis Bergmans & Asteraceae & Hemicryptophyte & Asian & Exotic \\
\hline Carduus pycnocephalus L. & Asteraceae & Terophyte & Eurasian & Exotic \\
\hline Centaurea melitensis $\mathrm{L}$. & Asteraceae & Terophyte & Mediterranean & Exotic \\
\hline Centaurea solstitialis L. & Asteraceae & Terophyte & Eurasian & Exotic \\
\hline Cichorium intybus L. & Asteraceae & Hemicryptophyte & Eurasian & Exotic \\
\hline Cirsium vulgare (Savi) Ten. & Asteraceae & Hemicryptophyte & Eurasian & Exotic \\
\hline $\begin{array}{l}\text { Cotula australis (Sieber ex Spreng.) } \\
\text { Hook. f. }\end{array}$ & Asteraceae & Terophyte & African & Exotic \\
\hline Cotula coronopifolia $\mathrm{L}$. & Asteraceae & Terophyte & African & Exotic \\
\hline Cynara cardunculus L. & Asteraceae & Hemicryptophyte & Mediterranean & Exotic \\
\hline Erigeron bonariensis L. & Asteraceae & Hemicryptophyte & American & Exotic \\
\hline Erigeron karwinskianus DC. & Asteraceae & Hemicryptophyte & American & Exotic \\
\hline Erigeron floribundus (Kunth) Sch. Bip. & Asteraceae & Terophyte & South American & Native \\
\hline $\begin{array}{l}\text { Euryops chrysanthemoides (DC.) B. } \\
\text { Nord. }\end{array}$ & Asteraceae & Nanophanerophyte & African & Exotic \\
\hline Euryops pectinatus (L.) Cass. & Asteraceae & Nanophanerophyte & African & Exotic \\
\hline Felicia amelloides (L.) Voss & Asteraceae & Chamaephyte & African & Exotic \\
\hline Galinsoga parviflora Cav. & Asteraceae & Terophyte & American & Exotic \\
\hline Gazania rigens (L.) Gaertn. & Asteraceae & Hemicryptophyte & African & Exotic \\
\hline Glebionis coronaria (L.) Cass. ex Spach & Asteraceae & Terophyte & Eurasian & Exotic \\
\hline Helianthus annuus L. & Asteraceae & Terophyte & American & Exotic \\
\hline Helianthus tuberosus L. & Asteraceae & Hemicryptophyte & American & Exotic \\
\hline Helminthotheca echioides (L.) Holub & Asteraceae & Terophyte & Eurasian & Exotic \\
\hline
\end{tabular}


Gayana Bot. 73(1), 2016

\begin{tabular}{|c|c|c|c|c|}
\hline Species & FAMILY & LIFE FORM & Native distribution & ORIGIN STATUS \\
\hline Hypochaeris radicata $\mathrm{L}$. & Asteraceae & Hemicryptophyte & Eurasian & Exotic \\
\hline $\begin{array}{l}\text { Jacobaea maritima (L.) Pelser \& } \\
\text { Meijden }\end{array}$ & Asteraceae & Nanophanerophyte & African & Exotic \\
\hline Lactuca serriola $\mathrm{L}$. & Asteraceae & Terophyte & Eurasian & Exotic \\
\hline Lactuca sativa $\mathrm{L}$. & Asteraceae & Terophyte & Eurasian & Exotic \\
\hline Lactuca virosa $\mathrm{L}$. & Asteraceae & Terophyte & Mediterranean & Exotic \\
\hline Matricaria chamomilla L. & Asteraceae & Terophyte & Eurasian & Exotic \\
\hline $\begin{array}{l}\text { Matricaria matricarioides (Less.) Porter } \\
\text { ex Britton }\end{array}$ & Asteraceae & Terophyte & $\begin{array}{l}\text { Eurasian and North } \\
\text { American }\end{array}$ & Exotic \\
\hline Osteospermum ecklonis (DC.) Norl. & Asteraceae & Hemicryptophyte & African & Exotic \\
\hline Osteospermum fruticosum (L.) Norl. & Asteraceae & Hemicryptophyte & African & Exotic \\
\hline Petasites fragans (Vill) C. Presl & Asteraceae & Hemicryptophyte & Eurasian & Exotic \\
\hline Santolina chamaecyparissus L. & Asteraceae & Nanophanerophyte & European & Exotic \\
\hline Senecio angulatus L. $f$. & Asteraceae & Nanophanerophyte & African & Exotic \\
\hline Senecio vulgaris $\mathrm{L}$. & Asteraceae & Terophyte & Eurasian & Exotic \\
\hline Silybum marianum (L.) Gaertn. & Asteraceae & Terophyte & Eurasian & Exotic \\
\hline Soliva sessilis Ruiz \& Pav. & Asteraceae & Terophyte & South American & Native \\
\hline Sonchus asper (L.) Hill & Asteraceae & Terophyte & Eurasian & Exotic \\
\hline Sonchus oleraceus (L.) L. & Asteraceae & Terophyte & Eurasian & Exotic \\
\hline Sonchus tenerrimus L. & Asteraceae & Terophyte & Eurasian & Exotic \\
\hline Tanacetum parthenium (L.) Sch. Bip. & Asteraceae & Hemicryptophyte & Eurasian & Exotic \\
\hline Taraxacum campylodes G. E. Haglund & Asteraceae & Hemicryptophyte & Eurasian & Exotic \\
\hline $\begin{array}{l}\text { Tessaria absinthioides (Hook. \& Arn.) } \\
\text { DC. }\end{array}$ & Asteraceae & Nanophanerophyte & South American & Native \\
\hline Berberis aquifolium Pursh & Berberidaceae & Nanophanerophyte & American & Exotic \\
\hline Berberis darwinii Hook. & Berberidaceae & Nanophanerophyte & South American & Native \\
\hline Berberis thunbergii DC. & Berberidaceae & Nanophanerophyte & Asian & Exotic \\
\hline Nandina domestica Thunb. & Berberidaceae & Nanophanerophyte & Asian & Exotic \\
\hline Betula pendula Roth & Betulaceae & Phanerophyte & Eurasian & Exotic \\
\hline Catalpa bignonioides Walter & Bignoniaceae & Phanerophyte & American & Exotic \\
\hline Jacaranda mimosifolia D. Don & Bignoniaceae & Phanerophyte & American & Exotic \\
\hline Amsinckia calycina (Moris) Chater & Boraginaceae & Terophyte & South American & Native \\
\hline Brassica napus L. & Brassicaceae & Terophyte & European & Exotic \\
\hline Brassica nigra (L.) K.Koch & Brassicaceae & Terophyte & Eurasian & Exotic \\
\hline Brassica rapa $\mathrm{L}$. & Brassicaceae & Terophyte & Mediterranean & Exotic \\
\hline Capsella bursa-pastoris (L.) Medik. & Brassicaceae & Terophyte & Mediterranean & Exotic \\
\hline Cardamine hirsuta $\mathrm{L}$. & Brassicaceae & Terophyte & European & Exotic \\
\hline Hirschfeldia incana (L.) Lagr.-Fossat & Brassicaceae & Terophyte & Eurasian & Exotic \\
\hline Lepidium didymum $\mathrm{L}$. & Brassicaceae & Terophyte & American & Exotic \\
\hline Lepidium draba $\mathrm{L}$. & Brassicaceae & Terophyte & African & Exotic \\
\hline Lepidium strictum (S. Watson) Rattan & Brassicaceae & Terophyte & American & Exotic \\
\hline Lobularia maritima (L.) Desv. & Brassicaceae & Hemicryptophyte & Mediterranean & Exotic \\
\hline Raphanus raphanistrum $\mathrm{L}$ & Brassicaceae & Terophyte & Mediterranean & Exotic \\
\hline Rapistrum rugosum (L.) All. & Brassicaceae & Terophyte & Eurasian & Exotic \\
\hline Sisymbrium irio L. & Brassicaceae & Terophyte & Eurasian & Exotic \\
\hline Sisymbrium officinale (L.) Scop. & Brassicaceae & Terophyte & Eurasian & Exotic \\
\hline
\end{tabular}




\begin{tabular}{|c|c|c|c|c|}
\hline SPECIES & FAMILY & LIFE FORM & NATIVE Distribution & ORIGIN STATUS \\
\hline Sisymbrium orientale L. & Brassicaceae & Terophyte & Eurasian & Exotic \\
\hline Buxus sempervirens L. & Buxaceae & Chamaephyte & Eurasian & Exotic \\
\hline $\begin{array}{l}\text { Austrocylindropuntia subulata } \\
\text { (Muehlenpf.) Backeb. }\end{array}$ & Cactaceae & Chamaephyte & American & Exotic \\
\hline $\begin{array}{l}\text { Echinopsis pachanoi (Britton \& Rose) } \\
\text { Friedrich \& G.D. Rowley }\end{array}$ & Cactaceae & Nanophanerophyte & American & Exotic \\
\hline Opuntia ficus-indica (L.) Mill. & Cactaceae & Nanophanerophyte & American & Exotic \\
\hline Cannabis sativa $\mathrm{L}$. & Cannabaceae & Terophyte & Asian & Exotic \\
\hline Celtis australis $\mathrm{L}$. & Cannabaceae & Phanerophyte & Mediterranean & Exotic \\
\hline Canna indica $\mathrm{L}$. & Cannaceae & Hemicryptophyte & American & Exotic \\
\hline $\begin{array}{l}\text { Abelia floribunda (M. Martens \& } \\
\text { Galeotti) Decne. }\end{array}$ & Caprifoliaceae & Phanerophyte & American & Exotic \\
\hline Abelia triflora R.Br. ex Wall. & Caprifoliaceae & Nanophanerophyte & Asian & Exotic \\
\hline Centranthus ruber (L.) DC. & Caprifoliaceae & Hemicryptophyte & Eurasian & Exotic \\
\hline Cerastium glomeratum Thuill. & Caryophyllaceae & Terophyte & Eurasian & Exotic \\
\hline Dianthus caryophyllus L. & Caryophyllaceae & Hemicryptophyte & Mediterranean & Exotic \\
\hline Polycarpon tetraphyllum (L.) L. & Caryophyllaceae & Terophyte & Eurasian & Exotic \\
\hline Sagina apetala Ard. & Caryophyllaceae & Terophyte & Eurasian & Exotic \\
\hline Spergularia media (L.) C.Presl & Caryophyllaceae & Terophyte & Eurasian & Exotic \\
\hline $\begin{array}{l}\text { Spergularia rubra (L.) J. Presl \& C. } \\
\text { Presl }\end{array}$ & Caryophyllaceae & Terophyte & Eurasian & Exotic \\
\hline Stellaria media (L.) Vill. & Caryophyllaceae & Terophyte & Eurasian & Exotic \\
\hline Casuarina cunninghamiana Miq. & Casuarinaceae & Phanerophyte & Oceanian and Asian & Exotic \\
\hline Euonymus japonicus Thunb. & Celastraceae & Nanophanerophyte & Asian & Exotic \\
\hline Maytenus boaria Molina & Celastraceae & Phanerophyte & South American & Native \\
\hline $\begin{array}{l}\text { Tradescantia } \times \text { andersoniana W. Ludw. } \\
\& \text { Rohweder }\end{array}$ & Commelinaceae & Chamaephyte & Artificial hybrid & Exotic \\
\hline Convolvulus arvensis $\mathrm{L}$. & Convolvulaceae & Hemicryptophyte & Eurasian & Exotic \\
\hline Dichondra sericea Sw. & Convolvulaceae & Hemicryptophyte & South American & Native \\
\hline Crassula arborescens (Mill.) Willd. & Crassulaceae & Nanophanerophyte & African & Exotic \\
\hline Crassula ovata (Mill.) Druce & Crassulaceae & Nanophanerophyte & African & Exotic \\
\hline $\begin{array}{l}\text { Cedrus deodara (Roxb. ex D. Don) G. } \\
\text { Don }\end{array}$ & Cupressaceae & Phanerophyte & Asian & Exotic \\
\hline Cedrus libanii A.Rich. & Cupressaceae & Phanerophyte & Asian & Exotic \\
\hline Cupressus funebris Endl. & Cupressaceae & Phanerophyte & Asian & Exotic \\
\hline Cupressus macrocarpa Hartw. & Cupressaceae & Phanerophyte & American & Exotic \\
\hline Cupressus sempervirens L. & Cupressaceae & Phanerophyte & Mediterranean & Exotic \\
\hline Platycladus orientalis (L.) Franco & Cupressaceae & Phanerophyte & Asian & Exotic \\
\hline Sequoia sempervirens (D.Don) Endl. & Cupressaceae & Phanerophyte & American & Exotic \\
\hline Taxodium distichum (L.) Rich. & Cupressaceae & Phanerophyte & American & Exotic \\
\hline Cyperus alternifolius $\mathrm{L}$. & Cyperaceae & Hemicryptophyte & African & Exotic \\
\hline Cyperus eragrostis Lam. & Cyperaceae & Hemicryptophyte & South American & Native \\
\hline Cyperus papyrus $\mathrm{L}$. & Cyperaceae & Hemicryptophyte & African & Exotic \\
\hline Elaeagnus angustifolia L. & Elaeagnaceae & Phanerophyte & Asian & Exotic \\
\hline Aristotelia chilensis (Molina) Stuntz & Elaeocarpaceae & Phanerophyte & South American & Native \\
\hline Crinodendron patagua Molina & Elaeocarpaceae & Phanerophyte & South American & Native \\
\hline Arbutus unedo L. & Ericaceae & Phanerophyte & Mediterranean & Exotic \\
\hline
\end{tabular}


Gayana Bot. 73(1), 2016

\begin{tabular}{|c|c|c|c|c|}
\hline SPECIES & FAMILY & LIFE FORM & Native distribution & ORIGIN STATUS \\
\hline Escallonia rubra (Ruiz \& Pav.) Pers. & Escalloniaceae & Nanophanerophyte & South American & Native \\
\hline Colliguaja odorifera Molina & Euphorbiaceae & Nanophanerophyte & South American & Native \\
\hline Euphorbia helioscopia L. & Euphorbiaceae & Terophyte & Eurasian & Exotic \\
\hline Euphorbia lathyris L. & Euphorbiaceae & Terophyte & Asian & Exotic \\
\hline Euphorbia peplus L. & Euphorbiaceae & Terophyte & Eurasian & Exotic \\
\hline $\begin{array}{l}\text { Euphorbia pulcherrima Willd. ex } \\
\text { Klotzsch }\end{array}$ & Euphorbiaceae & Nanophanerophyte & American & Exotic \\
\hline Euphorbia serpens Kunth & Euphorbiaceae & Terophyte & South American & Native \\
\hline Ricinus communis L. & Euphorbiaceae & Nanophanerophyte & African & Exotic \\
\hline Acacia caven (Molina) Molina & Fabaceae & Phanerophyte & South American & Native \\
\hline Acacia dealbata Link & Fabaceae & Phanerophyte & Oceanian & Exotic \\
\hline Acacia karroo Hayne & Fabaceae & Phanerophyte & African & Exotic \\
\hline Acacia melanoxylon R. Br. & Fabaceae & Phanerophyte & Oceanian & Exotic \\
\hline Acacia saligna (Labill.) Wendl. & Fabaceae & Phanerophyte & Oceanian & Exotic \\
\hline Acacia visco Griseb. & Fabaceae & Phanerophyte & American & Exotic \\
\hline Albizia julibrissin Durazz. & Fabaceae & Phanerophyte & Asian & Exotic \\
\hline Bauhinia forficata Link & Fabaceae & Phanerophyte & American & Exotic \\
\hline $\begin{array}{l}\text { Caesalpinia gilliesii (Wall. ex Hook.) } \\
\text { D. Dietr. }\end{array}$ & Fabaceae & Nanophanerophyte & American & Exotic \\
\hline Caesalpinia spinosa (Molina) Kuntze & Fabaceae & Phanerophyte & South American & Native \\
\hline Cercis siliquastrum L. & Fabaceae & Phanerophyte & Eurasian & Exotic \\
\hline Erythrina crista-galli L. & Fabaceae & Phanerophyte & American & Exotic \\
\hline Erythrina falcata Benth. & Fabaceae & Phanerophyte & American & Exotic \\
\hline Galega officinalis L. & Fabaceae & Hemicryptophyte & Eurasian & Exotic \\
\hline $\begin{array}{l}\text { Genista monspessulana (L.) L.A.S. } \\
\text { Johnson }\end{array}$ & Fabaceae & Nanophanerophyte & Mediterranean & Exotic \\
\hline Gleditsia triacanthos L. & Fabaceae & Phanerophyte & American & Exotic \\
\hline Lotus tenuis Waldst. \& Kit. & Fabaceae & Terophyte & American & Exotic \\
\hline Lupinus polyphyllus Lindl. & Fabaceae & Hemicryptophyte & American & Exotic \\
\hline Medicago lupulina $\mathrm{L}$. & Fabaceae & Terophyte & Eurasian & Exotic \\
\hline Medicago polymorpha $\mathrm{L}$. & Fabaceae & Terophyte & Eurasian & Exotic \\
\hline Medicago sativa $\mathrm{L}$. & Fabaceae & Chamaephyte & Eurasian & Exotic \\
\hline Melilotus albus Medik. & Fabaceae & Terophyte & European & Exotic \\
\hline Melilotus indicus (L.) All. & Fabaceae & Terophyte & Eurasian & Exotic \\
\hline Parkinsonia aculeata L. & Fabaceae & Phanerophyte & American & Exotic \\
\hline Prosopis chilensis (Molina) Stuntz & Fabaceae & Phanerophyte & South American & Native \\
\hline Robinia hispida L. & Fabaceae & Phanerophyte & American & Exotic \\
\hline Robinia pseudoacacia L. & Fabaceae & Phanerophyte & American & Exotic \\
\hline $\begin{array}{l}\text { Senna candolleana (Vogel) H.S.Irwin \& } \\
\text { Barneby }\end{array}$ & Fabaceae & Phanerophyte & South American & Native \\
\hline Sophora cassioides (Phil.) Sparre & Fabaceae & Phanerophyte & South American & Native \\
\hline Sophora macrocarpa $\mathrm{Sm}$. & Fabaceae & Nanophanerophyte & South American & Native \\
\hline Spartium junceum L. & Fabaceae & Nanophanerophyte & Mediterranean & Exotic \\
\hline Styphnolobium japonicum (L.) Schott & Fabaceae & Phanerophyte & Asian & Exotic \\
\hline Trifolium fragiferum $\mathrm{L}$. & Fabaceae & Hemicryptophyte & Eurasian & Exotic \\
\hline Trifolium repens $\mathrm{L}$. & Fabaceae & Hemicryptophyte & Eurasian & Exotic \\
\hline
\end{tabular}


Urban flora of Santiago: FigueroA, J.A. ET AL.

\begin{tabular}{|c|c|c|c|c|}
\hline SPECIES & FAMILY & LIFE FORM & Native distribution & ORIGIN STATUS \\
\hline Vicia sativa $\mathrm{L}$. & Fabaceae & Terophyte & Eurasian & Exotic \\
\hline Wisteria sinensis (Sims) Sweet & Fabaceae & Nanophanerophyte & Asian & Exotic \\
\hline Castanea sativa Mill. & Fagaceae & Phanerophyte & European & Exotic \\
\hline Quercus ilex L. & Fagaceae & Phanerophyte & Eurasian & Exotic \\
\hline Quercus nigra L. & Fagaceae & Phanerophyte & American & Exotic \\
\hline Quercus robur L. & Fagaceae & Phanerophyte & Eurasian & Exotic \\
\hline Quercus suber L. & Fagaceae & Phanerophyte & Mediterranean & Exotic \\
\hline Erodium botrys (Cav.) Bertol. & Geraniaceae & Terophyte & European & Exotic \\
\hline Erodium cicutarium (L.) L’Hér. & Geraniaceae & Terophyte & Eurasian & Exotic \\
\hline Erodium malacoides (L.) L’Hér. & Geraniaceae & Terophyte & Eurasian & Exotic \\
\hline Erodium moschatum (L.) L'Hér. & Geraniaceae & Terophyte & Eurasian & Exotic \\
\hline Geranium dissectum $\mathrm{L}$. & Geraniaceae & Terophyte & European & Exotic \\
\hline Geranium robertianum L. & Geraniaceae & Terophyte & Eurasian & Exotic \\
\hline Pelargonium graveolens L’Hér. & Geraniaceae & Nanophanerophyte & African & Exotic \\
\hline Pelargonium $\times$ hortorum L.H. Bailey & Geraniaceae & Chamaephyte & Artificial hybrid & Exotic \\
\hline Pelargonium zonale (L.) L'Hér. ex Aiton & Geraniaceae & Chamaephyte & African & Exotic \\
\hline Ginkgo biloba L. & Ginkgoaceae & Phanerophyte & Asian & Exotic \\
\hline Hydrangea macrophylla (Thunb.) Ser. & Hydrangeaceae & Chamaephyte & Asian & Exotic \\
\hline Philadelphus coronarius L. & Hydrangeaceae & Nanophanerophyte & European & Exotic \\
\hline Hypericum calycinum $\mathrm{L}$. & Hypericaceae & Chamaephyte & Eurasian & Exotic \\
\hline Hypericum perforatum $\mathrm{L}$. & Hypericaceae & Chamaephyte & Eurasian & Exotic \\
\hline Chasmanthe aethiopica (L.) N.E.Br. & Iridaceae & Hemicryptophyte & African & Exotic \\
\hline $\begin{array}{l}\text { Crocosmia } \times \text { crocosmiiflora (Lemoine) } \\
\text { N.E. Br. }\end{array}$ & Iridaceae & Hemicryptophyte & Artificial hybrid & Exotic \\
\hline Iris orientalis Mill. & Iridaceae & Hemicryptophyte & Mediterranean & Exotic \\
\hline Iris $\times$ germanica $\mathrm{L}$. & Iridaceae & Hemicryptophyte & Artificial hybrid & Exotic \\
\hline Sisyrinchium striatum Dm. & Iridaceae & Hemicryptophyte & South American & Native \\
\hline Juglans regia $\mathrm{L}$. & Juglandaceae & Phanerophyte & Eurasian & Exotic \\
\hline Ajuga reptans L. & Lamiaceae & Hemicryptophyte & European & Exotic \\
\hline Lamium amplexicaule $\mathrm{L}$. & Lamiaceae & Terophyte & Eurasian & Exotic \\
\hline Lamium galeobdolon (L.) L. & Lamiaceae & Terophyte & Eurasian & Exotic \\
\hline Lavandula angustifolia Mill. & Lamiaceae & Nanophanerophyte & European & Exotic \\
\hline Marrubium vulgare L. & Lamiaceae & Hemicryptophyte & Eurasian & Exotic \\
\hline Mentha $\times$ piperita $\mathrm{L}$. & Lamiaceae & Hemicryptophyte & European & Exotic \\
\hline Mentha pulegium L. & Lamiaceae & Hemicryptophyte & Eurasian & Exotic \\
\hline Origanum majorana L. & Lamiaceae & Hemicryptophyte & Asian & Exotic \\
\hline Rosmarinus officinalis L. & Lamiaceae & Nanophanerophyte & Mediterranean & Exotic \\
\hline Salvia leucantha Cav. & Lamiaceae & Nanophanerophyte & American & Exotic \\
\hline Salvia microphylla Kunth & Lamiaceae & Nanophanerophyte & African & Exotic \\
\hline Salvia officinalis L. & Lamiaceae & Nanophanerophyte & Mediterranean & Exotic \\
\hline Stachys palaestina $\mathrm{L}$. & Lamiaceae & Chamaephyte & African and Asian & Exotic \\
\hline Teucrium fruticans $\mathrm{L}$. & Lamiaceae & Nanophanerophyte & African and Asian & Exotic \\
\hline $\begin{array}{l}\text { Beilschmiedia berteroana (Gay) } \\
\text { Kosterm. }\end{array}$ & Lauraceae & Phanerophyte & South American & Native \\
\hline Beilschmiedia miersii (Gay) Kosterm. & Lauraceae & Phanerophyte & South American & Native \\
\hline Cryptocarya alba (Molina) Looser & Lauraceae & Phanerophyte & South American & Native \\
\hline
\end{tabular}


Gayana Bot. 73(1), 2016

\begin{tabular}{|c|c|c|c|c|}
\hline SpecIES & FAMILY & LIFE FORM & Native Distribution & ORIGIN STATUS \\
\hline Laurus nobilis L. & Lauraceae & Phanerophyte & Mediterranean & Exotic \\
\hline Persea americana Mill. & Lauraceae & Phanerophyte & American & Exotic \\
\hline Loasa triloba Dombey ex Juss. & Loasaceae & Terophyte & South American & Native \\
\hline Tristerix corymbosus (L.) Kuijt & Loranthaceae & Phanerophyte & South American & Native \\
\hline Lagerstroemia indica $\mathrm{L}$. & Lythraceae & Phanerophyte & Asian & Exotic \\
\hline Punica granatum $\mathrm{L}$. & Lythraceae & Nanophanerophyte & Mediterranean & Exotic \\
\hline Liriodendron tulipifera L. & Magnoliaceae & Phanerophyte & North American & Exotic \\
\hline Magnolia grandiflora $\mathrm{L}$. & Magnoliaceae & Phanerophyte & American & Exotic \\
\hline Magnolia $\times$ soulangeana Soul.-Bod. & Magnoliaceae & Nanophanerophyte & Artificial hybrid & Exotic \\
\hline Abutilon $\times$ hybridum Voss & Malvaceae & Nanophanerophyte & Artificial hybrid & Exotic \\
\hline Alcea rosea $\mathrm{L}$. & Malvaceae & Terophyte & Mediterranean & Exotic \\
\hline $\begin{array}{l}\text { Brachychiton acerifolius (A.Cunn. ex } \\
\text { G.Don) F.Muell. }\end{array}$ & Malvaceae & Phanerophyte & Oceanian & Exotic \\
\hline Brachychiton discolor F. Muell. & Malvaceae & Phanerophyte & Oceanian & Exotic \\
\hline $\begin{array}{l}\text { Brachychiton populneus (Schott \& } \\
\text { Endl.) R.Br. }\end{array}$ & Malvaceae & Phanerophyte & Oceanian & Exotic \\
\hline Hibiscus rosa-sinensis L. & Malvaceae & Nanophanerophyte & Asian & Exotic \\
\hline $\begin{array}{l}\text { Lagunaria patersonia (Andrews) G. } \\
\text { Don }\end{array}$ & Malvaceae & Phanerophyte & Oceanian & Exotic \\
\hline Malva neglecta Wallr. & Malvaceae & Terophyte & Eurasian & Exotic \\
\hline Malva nicaensis All. & Malvaceae & Terophyte & Eurasian & Exotic \\
\hline Malva parviflora $\mathrm{L}$. & Malvaceae & Terophyte & Eurasian & Exotic \\
\hline Malva sylvestris $\mathrm{L}$. & Malvaceae & Terophyte & Eurasian & Exotic \\
\hline Malvella leprosa (Ortega) Krapov. & Malvaceae & Terophyte & South American & Native \\
\hline Modiola caroliniana (L.) G.Don & Malvaceae & Hemicryptophyte & South American & Native \\
\hline Sphaeralcea obtusiloba G. Don & Malvaceae & Chamaephyte & South American & Native \\
\hline Tilia americana $\mathrm{L}$. & Malvaceae & Phanerophyte & American & Exotic \\
\hline Melia azedarach $\mathrm{L}$. & Meliaceae & Phanerophyte & Asian & Exotic \\
\hline Peumus boldus Molina & Monimiaceae & Phanerophyte & South American & Native \\
\hline Ficus carica L. & Moraceae & Phanerophyte & Eurasian & Exotic \\
\hline Ficus elastica Roxb. ex Hornem. & Moraceae & Phanerophyte & Asian & Exotic \\
\hline Ficus repens Roxb. ex Sm. & Moraceae & Phanerophyte & Asian & Exotic \\
\hline Morus alba L. & Moraceae & Phanerophyte & Asian & Exotic \\
\hline Morus nigra $\mathrm{L}$. & Moraceae & Phanerophyte & Asian & Exotic \\
\hline Eucalyptus globulus Labill. & Myrtaceae & Phanerophyte & Oceanian & Exotic \\
\hline Luma apiculata (DC.) Burret & Myrtaceae & Phanerophyte & South American & Native \\
\hline Luma chequen (Molina) A.Gray & Myrtaceae & Nanophanerophyte & South American & Native \\
\hline $\begin{array}{l}\text { Myrceugenia lanceolata (Juss. ex J. St.- } \\
\text { Hil.) Kausel }\end{array}$ & Myrtaceae & Nanophanerophyte & South American & Native \\
\hline Myrtus communis L. & Myrtaceae & Nanophanerophyte & Mediterranean & Exotic \\
\hline Mirabilis jalapa $\mathrm{L}$. & Nyctaginaceae & Hemicryptophyte & American & Exotic \\
\hline Fraxinus excelsior L. & Oleaceae & Phanerophyte & Asian & Exotic \\
\hline Fraxinus ornus L. & Oleaceae & Phanerophyte & Eurasian & Exotic \\
\hline Fraxinus pennsylvanica Marshall & Oleaceae & Phanerophyte & American & Exotic \\
\hline Jasminum mesnyi Hance & Oleaceae & Nanophanerophyte & Asian & Exotic \\
\hline Jasminum nudiflorum Lindl. & Oleaceae & Nanophanerophyte & Asian & Exotic \\
\hline
\end{tabular}


Urban flora of Santiago: FigueroA, J.A. ET AL.

\begin{tabular}{|c|c|c|c|c|}
\hline Species & FAMILY & LIFE FORM & NATIVE DistRibution & ORIGIN STATUS \\
\hline Jasminum officinale L. & Oleaceae & Nanophanerophyte & Asian & Exotic \\
\hline Jasminum polyanthum Franch. & Oleaceae & Nanophanerophyte & Asian & Exotic \\
\hline Ligustrum japonicum Thunb. & Oleaceae & Nanophanerophyte & Asian & Exotic \\
\hline Ligustrum lucidum W.T. Aiton & Oleaceae & Phanerophyte & Asian & Exotic \\
\hline Ligustrum ovalifolium Hassk. & Oleaceae & Nanophanerophyte & Asian & Exotic \\
\hline Ligustrum sinense Lour. & Oleaceae & Nanophanerophyte & Asian & Exotic \\
\hline Olea europaea L. & Oleaceae & Phanerophyte & Mediterranean & Exotic \\
\hline Syringa vulgaris $\mathrm{L}$. & Oleaceae & Nanophanerophyte & Mediterranean & Exotic \\
\hline Camissonia dentata (Cav.) Reiche & Onagraceae & Terophyte & South American & Native \\
\hline $\begin{array}{l}\text { Clarkia tenella (Cav.) H.F.Lewis \& } \\
\text { M.R.Lewis }\end{array}$ & Onagraceae & Terophyte & South American & Native \\
\hline Epilobium ciliatum Raf. & Onagraceae & Hemicryptophyte & South American & Native \\
\hline $\begin{array}{l}\text { Fuchsia } \times \text { hybrida hort. ex Siebert \& } \\
\text { Voss }\end{array}$ & Onagraceae & Nanophanerophyte & Artificial hybrid & Exotic \\
\hline Fuchsia magellanica Lam. & Onagraceae & Nanophanerophyte & South American & Native \\
\hline Oxalis arenaria Bertero & Oxalidaceae & Geophyte & South American & Native \\
\hline Oxalis articulata Savigny & Oxalidaceae & Hemicryptophyte & American & Exotic \\
\hline Oxalis corniculata L. & Oxalidaceae & Chamaephyte & African & Exotic \\
\hline Oxalis incarnata $\mathrm{L}$. & Oxalidaceae & Hemicryptophyte & African & Exotic \\
\hline Oxalis pes-caprae $\mathrm{L}$. & Oxalidaceae & Hemicryptophyte & African & Exotic \\
\hline Oxalis rosea Jacq. & Oxalidaceae & Terophyte & South American & Native \\
\hline Oxalis triangularis A. St.-Hil. & Oxalidaceae & Hemicryptophyte & American & Exotic \\
\hline Eschscholzia californica Cham. & Papaveraceae & Hemicryptophyte & American & Exotic \\
\hline Fumaria agraria Lag. & Papaveraceae & Terophyte & Eurasian & Exotic \\
\hline Fumaria capreolata L. & Papaveraceae & Terophyte & Eurasian & Exotic \\
\hline Fumaria officinalis L. & Papaveraceae & Terophyte & European & Exotic \\
\hline Papaver somniferum L. & Papaveraceae & Terophyte & African and Asian & Exotic \\
\hline Passiflora caerulea L. & Passifloraceae & Geophyte & American & Exotic \\
\hline Paulownia tomentosa Steud. & Paulowniaceae & Phanerophyte & Asian & Exotic \\
\hline Phytolacca dioica $\mathrm{L}$. & Phytolaccaceae & Phanerophyte & American & Exotic \\
\hline Abies procera Rehder & Pinaceae & Phanerophyte & American & Exotic \\
\hline Pinus canariensis C.Sm. & Pinaceae & Phanerophyte & African & Exotic \\
\hline Pinus radiata D. Don & Pinaceae & Phanerophyte & American & Exotic \\
\hline Pseudotsuga menziesii (Mirb.) Franco & Pinaceae & Phanerophyte & American & Exotic \\
\hline $\begin{array}{l}\text { Pittosporum tenuifolium Banks \& Sol. } \\
\text { ex Gaertn }\end{array}$ & Pittosporaceae & Phanerophyte & Oceanian & Exotic \\
\hline Pittosporum tobira (Thunb.) W.T. Aiton & Pittosporaceae & Nanophanerophyte & Asian & Exotic \\
\hline Pittosporum undulatum Vent. & Pittosporaceae & Phanerophyte & Oceanian & Exotic \\
\hline Antirrhinum majus L. & Plantaginaceae & Terophyte & American & Exotic \\
\hline $\begin{array}{l}\text { Cymbalaria muralis P.Gaertn., B.Mey. } \\
\text { \& Scherb. }\end{array}$ & Plantaginaceae & Chamaephyte & European & Exotic \\
\hline Hebe buxifolia Cockayne \& Allan & Plantaginaceae & Nanophanerophyte & Oceanian & Exotic \\
\hline Hebe franciscana (Eastw.) Souster & Plantaginaceae & Nanophanerophyte & Artificial hybrid & Exotic \\
\hline Plantago lanceolata $\mathrm{L}$. & Plantaginaceae & Hemicryptophyte & Eurasian & Exotic \\
\hline Plantago major L. & Plantaginaceae & Hemicryptophyte & Eurasian & Exotic \\
\hline Veronica arvensis L. & Plantaginaceae & Terophyte & European & Exotic \\
\hline
\end{tabular}


Gayana Bot. 73(1), 2016

\begin{tabular}{|c|c|c|c|c|}
\hline Species & FAMILY & LIFE FORM & Native distribution & ORIGIN STATUS \\
\hline Veronica persica Poir. & Plantaginaceae & Terophyte & Eurasian & Exotic \\
\hline Platanus acerifolia (Aiton) Willd. & Platanaceae & Phanerophyte & Eurasian & Exotic \\
\hline Plumbago auriculata Lam. & Plumbaginaceae & Nanophanerophyte & African & Exotic \\
\hline $\begin{array}{l}\text { Amelichloa caudata (Trin.) Arriaga \& } \\
\text { Barkworth }\end{array}$ & Poaceae & Hemicryptophyte & South American & Native \\
\hline Anthoxanthum odoratum L. & Poaceae & Terophyte & Eurasian & Exotic \\
\hline Avena barbata Pott ex Link & Poaceae & Terophyte & African and Asian & Exotic \\
\hline Avena fatua $\mathrm{L}$. & Poaceae & Terophyte & Eurasian & Exotic \\
\hline Avena sativa $\mathrm{L}$. & Poaceae & Terophyte & Eurasian & Exotic \\
\hline Avena sterilis L. & Poaceae & Terophyte & Eurasian & Exotic \\
\hline Briza minor L. & Poaceae & Terophyte & Mediterranean & Exotic \\
\hline Bromus berteroanus Colla & Poaceae & Terophyte & South American & Native \\
\hline Bromus catharticus Vahl & Poaceae & Hemicryptophyte & South American & Native \\
\hline Bromus cebadilla Steudel & Poaceae & Hemicryptophyte & Oceanian & Exotic \\
\hline Bromus diandrus Roth & Poaceae & Terophyte & European & Exotic \\
\hline Bromus hordeaceus L. & Poaceae & Terophyte & Eurasian & Exotic \\
\hline Bromus madritensis L. & Poaceae & Terophyte & Eurasian & Exotic \\
\hline Bromus racemosus $\mathrm{L}$. & Poaceae & Terophyte & Eurasian & Exotic \\
\hline Bromus scoparius L. & Poaceae & Terophyte & Mediterranean & Exotic \\
\hline $\begin{array}{l}\text { Chaetotropis elongata (Kunth) } \\
\text { Björkman }\end{array}$ & Poaceae & Hemicryptophyte & South American & Native \\
\hline $\begin{array}{l}\text { Cortaderia selloana (Schult. \& Schult. } \\
\text { f.) Asch. \& Graebn. }\end{array}$ & Poaceae & Hemicryptophyte & American & Exotic \\
\hline Cynodon dactylon (L.) Pers. & Poaceae & Hemicryptophyte & Eurasian & Exotic \\
\hline Dactylis glomerata L. & Poaceae & Hemicryptophyte & Eurasian & Exotic \\
\hline Echinochloa crus-galli (L.) P. Beauv. & Poaceae & Terophyte & Eurasian & Exotic \\
\hline Eleusine tristachya (Lam.) Lam. & Poaceae & Terophyte & $\begin{array}{l}\text { American and } \\
\text { African }\end{array}$ & Exotic \\
\hline Eragrostis virescens J.Presl & Poaceae & Terophyte & South American & Native \\
\hline Festuca acanthophylla É.Desv. & Poaceae & Hemicryptophyte & South American & Native \\
\hline Festuca arundinacea Schreb. & Poaceae & Hemicryptophyte & Eurasian & Exotic \\
\hline Festuca ovina Fr. & Poaceae & Hemicryptophyte & European & Exotic \\
\hline Festuca rubra L. & Poaceae & Hemicryptophyte & Eurasian & Exotic \\
\hline Holcus lanatus L. & Poaceae & Hemicryptophyte & Eurasian & Exotic \\
\hline Hordeum murinum L. & Poaceae & Terophyte & Eurasian & Exotic \\
\hline Lolium multiflorum Lam. & Poaceae & Terophyte & African and Asian & Exotic \\
\hline Lolium perenne L. & Poaceae & Hemicryptophyte & Eurasian & Exotic \\
\hline Nassella laevissima (Phil.) Barkworth & Poaceae & Hemicryptophyte & South American & Native \\
\hline Nassella tenuissima (Trin.) Barkworth & Poaceae & Hemicryptophyte & South American & Native \\
\hline Piptatherum miliaceum (L.) Coss. & Poaceae & Hemicryptophyte & Eurasian & Exotic \\
\hline $\begin{array}{l}\text { Pennisetum clandestinum Hochst. ex } \\
\text { Chiov. }\end{array}$ & Poaceae & Hemicryptophyte & African & Exotic \\
\hline Pennisetum purpureum Schumach. & Poaceae & Hemicryptophyte & African & Exotic \\
\hline Pennisetum setaceum (Forssk.) Chiov. & Poaceae & Hemicryptophyte & African & Exotic \\
\hline Pennisetum villosum Fresen. & Poaceae & Hemicryptophyte & African and Asian & Exotic \\
\hline Phalaris canariensis L. & Poaceae & Hemicryptophyte & Mediterranean & Exotic \\
\hline
\end{tabular}


Urban flora of Santiago: FigueroA, J.A. ET AL.

\begin{tabular}{|c|c|c|c|c|}
\hline Species & FAMILY & LIFE FORM & NATIVE Distribution & ORIGIN STATUS \\
\hline $\begin{array}{l}\text { Phyllostachys aurea Rivière \& C. } \\
\text { Rivière }\end{array}$ & Poaceae & Hemicryptophyte & Asian & Exotic \\
\hline $\begin{array}{l}\text { Piptochaetium stipoides (Trin. \& Rupr.) } \\
\text { Hack. \& Arechav. }\end{array}$ & Poaceae & Hemicryptophyte & South American & Native \\
\hline Poa annua $\mathrm{L}$. & Poaceae & Terophyte & European & Exotic \\
\hline Poa pratensis L. & Poaceae & Hemicryptophyte & Eurasian & Exotic \\
\hline Polypogon australis Brongn. & Poaceae & Hemicryptophyte & South American & Native \\
\hline Polypogon linearis Trin. & Poaceae & Hemicryptophyte & South American & Native \\
\hline Polypogon viridis (Gouan) Breistr. & Poaceae & Terophyte & Eurasian & Exotic \\
\hline Pseudosasa japonica (Steud.) Makino & Poaceae & Hemicryptophyte & Asian & Exotic \\
\hline Rostraria cristata (L.) Tzvelev & Poaceae & Terophyte & Eurasian & Exotic \\
\hline Setaria parviflora (Poir.) M.Kerguelen & Poaceae & Terophyte & South American & Native \\
\hline Sorghum halepense (L.) Pers. & Poaceae & Hemicryptophyte & African and Asian & Exotic \\
\hline Sporobolus indicus (L.) R.Br. & Poaceae & Terophyte & South American & Native \\
\hline Sporobolus virginicus (L.) Kunth & Poaceae & Hemicryptophyte & South American & Native \\
\hline Triticum aestivum $\mathrm{L}$. & Poaceae & Terophyte & Asian & Exotic \\
\hline Vulpia myuros (L.) C.C.Gmel. & Poaceae & Terophyte & Eurasian & Exotic \\
\hline Zea mays L. & Poaceae & Terophyte & American & Exotic \\
\hline Cantua buxifolia Juss. ex Lam. & Polemoniaceae & Nanophanerophyte & American & Exotic \\
\hline $\begin{array}{l}\text { Muehlenbeckia hastulata (Sm.) I.M. } \\
\text { Johnst. }\end{array}$ & Polygonaceae & Nanophanerophyte & South American & Native \\
\hline Polygonum aviculare L. & Polygonaceae & Terophyte & European & Exotic \\
\hline Rumex acetosella $\mathrm{L}$. & Polygonaceae & Hemicryptophyte & European & Exotic \\
\hline Rumex pulcher L. & Polygonaceae & Hemicryptophyte & Mediterranean & Exotic \\
\hline Portulaca oleracea L. & Portulacaceae & Terophyte & Asian & Exotic \\
\hline Anagallis arvensis L. & Primulaceae & Terophyte & Eurasian & Exotic \\
\hline Primula vulgaris Huds. & Primulaceae & Hemicryptophyte & European & Exotic \\
\hline Grevillea robusta A. Cunn. ex R. Br. & Proteaceae & Phanerophyte & Eurasian & Exotic \\
\hline Quillaja saponaria Molina & Quillajaceae & Phanerophyte & South American & Native \\
\hline Consolida ajacis (L.) Schur & Ranunculaceae & Terophyte & European & Exotic \\
\hline Ranunculus muricatus L. & Ranunculaceae & Hemicryptophyte & Eurasian & Exotic \\
\hline Ceanothus caeruleus Lag. & Rhamnaceae & Nanophanerophyte & American & Exotic \\
\hline $\begin{array}{l}\text { Retanilla trinervia (Gillies \& Hook.) } \\
\text { Hook. \& Arn. }\end{array}$ & Rhamnaceae & Nanophanerophyte & South American & Native \\
\hline Rhamnus alaternus $\mathrm{L}$. & Rhamnaceae & Nanophanerophyte & Mediterranean & Exotic \\
\hline $\begin{array}{l}\text { Chaenomeles japonica (Thunb.) Lindl. } \\
\text { ex Spach }\end{array}$ & Rosaceae & Nanophanerophyte & Asian & Exotic \\
\hline Cotoneaster lacteus W. W. Sm. & Rosaceae & Nanophanerophyte & Asian & Exotic \\
\hline Cotoneaster horizontalis Decne. & Rosaceae & Nanophanerophyte & Eurasian & Exotic \\
\hline Cotoneaster pannosus Franch. & Rosaceae & Nanophanerophyte & Asian & Exotic \\
\hline Crataegus crus-galli $\mathrm{L}$. & Rosaceae & Nanophanerophyte & North American & Exotic \\
\hline Crataegus monogyna Jacq. & Rosaceae & Phanerophyte & Eurasian & Exotic \\
\hline Cydonia oblonga Mill. & Rosaceae & Phanerophyte & Asian & Exotic \\
\hline Eriobotrya japonica (Thunb.) Lindl. & Rosaceae & Phanerophyte & Asian & Exotic \\
\hline Fragaria chiloensis (L.) Mill. & Rosaceae & Chamaephyte & South American & Native \\
\hline Malus communis Desf. & Rosaceae & Phanerophyte & Eurasian & Exotic \\
\hline
\end{tabular}


Gayana Bot. 73(1), 2016

\begin{tabular}{|c|c|c|c|c|}
\hline SPECIES & FAMILY & LIFE FORM & Native distribution & ORIGIN STATUS \\
\hline Malus pumila Mill. & Rosaceae & Phanerophyte & European & Exotic \\
\hline Malus $\times$ purpurea (E.Barbier) Rehder & Rosaceae & Phanerophyte & Artificial hybrid & Exotic \\
\hline Photinia serrulata Lindl. & Rosaceae & Phanerophyte & Asian & Exotic \\
\hline Prunus armeniaca L. & Rosaceae & Phanerophyte & Asian & Exotic \\
\hline Prunus avium (L.) L. & Rosaceae & Phanerophyte & Mediterranean & Exotic \\
\hline Prunus cerasifera Ehrh. & Rosaceae & Phanerophyte & Eurasian & Exotic \\
\hline Prunus cerasus $\mathrm{L}$. & Rosaceae & Phanerophyte & Eurasian & Exotic \\
\hline Prunus domestica $\mathrm{L}$. & Rosaceae & Phanerophyte & Asian & Exotic \\
\hline Prunus dulcis (Mill.) D.A.Webb & Rosaceae & Phanerophyte & Mediterranean & Exotic \\
\hline $\begin{array}{l}\text { Prunus ilicifolia (Nutt. ex Hook. \& } \\
\text { Arn.) D. Dietr. }\end{array}$ & Rosaceae & Phanerophyte & American & Exotic \\
\hline Prunus laurocerasus L. & Rosaceae & Phanerophyte & Eurasian & Exotic \\
\hline Prunus persica (L.) Batsch & Rosaceae & Phanerophyte & Asian & Exotic \\
\hline Pyracantha coccinea M. Roem. & Rosaceae & Nanophanerophyte & Eurasian & Exotic \\
\hline Pyrus communis L. & Rosaceae & Phanerophyte & Eurasian & Exotic \\
\hline Rosa helenae Rehder \& E.H. Wilson & Rosaceae & Nanophanerophyte & Asian & Exotic \\
\hline Rosa $\times$ hybrida Schleich. & Rosaceae & Nanophanerophyte & Artificial hybrid & Exotic \\
\hline Rubus ulmifolius Schott & Rosaceae & Nanophanerophyte & European & Exotic \\
\hline Spiraea cantoniensis Lour. & Rosaceae & Nanophanerophyte & Asian & Exotic \\
\hline Spiraea japonica L.f. & Rosaceae & Nanophanerophyte & Asian & Exotic \\
\hline Spiraea thunbergii Siebold ex Blume & Rosaceae & Nanophanerophyte & Asian & Exotic \\
\hline Coprosma baueri Endl. & Rosaceae & Nanophanerophyte & Oceanian & Exotic \\
\hline Galium aparine $\mathrm{L}$. & Rubiaceae & Terophyte & Eurasian & Exotic \\
\hline Galium parisiense $\mathrm{L}$. & Rubiaceae & Terophyte & Eurasian & Exotic \\
\hline Citrus $\times$ aurantium $\mathrm{L}$. & Rutaceae & Phanerophyte & Asian & Exotic \\
\hline Citrus limon (L.) Osbeck & Rutaceae & Phanerophyte & Asian & Exotic \\
\hline Citrus reticulata Blanco & Rutaceae & Phanerophyte & Asian & Exotic \\
\hline Citrus sinensis (L.) Osbeck & Rutaceae & Phanerophyte & Asian & Exotic \\
\hline Ruta graveolens $\mathrm{L}$. & Rutaceae & Chamaephyte & European & Exotic \\
\hline Populus alba L. & Salicaceae & Phanerophyte & Eurasian & Exotic \\
\hline Populus deltoides Marshall & Salicaceae & Phanerophyte & American & Exotic \\
\hline Populus nigra L. & Salicaceae & Phanerophyte & Eurasian & Exotic \\
\hline Salix babylonica L. & Salicaceae & Phanerophyte & Asian & Exotic \\
\hline Acer negundo L. & Sapindaceae & Phanerophyte & American & Exotic \\
\hline Acer palmatum Thunb. & Sapindaceae & Phanerophyte & Asian & Exotic \\
\hline Acer pseudoplatanus L. & Sapindaceae & Phanerophyte & Eurasian & Exotic \\
\hline Aesculus hippocastanum L. & Sapindaceae & Phanerophyte & European & Exotic \\
\hline Aesculus $\times$ carnea Zeyh. & Sapindaceae & Phanerophyte & European & Exotic \\
\hline Koelreuteria paniculata Laxm. & Sapindaceae & Nanophanerophyte & Asian & Exotic \\
\hline Buddleja globosa Hope & Scrophulariaceae & Nanophanerophyte & South American & Native \\
\hline Myoporum laetum G. Forst. & Scrophulariaceae & Phanerophyte & Oceanian & Exotic \\
\hline Verbascum virgatum Stokes & Scrophulariaceae & Terophyte & European & Exotic \\
\hline Ailanthus altissima (Mill.) Swingle & Simaroubaceae & Phanerophyte & Asian & Exotic \\
\hline Brugmansia arborea (L.) Steud. & Solanaceae & Nanophanerophyte & American & Exotic \\
\hline Cestrum parqui (Lam.) L’Hér. & Solanaceae & Nanophanerophyte & South American & Native \\
\hline
\end{tabular}




\begin{tabular}{|c|c|c|c|c|}
\hline SpeCIES & FAMILY & LIFE FORM & Native Distribution & ORIGIN STATUS \\
\hline Datura ferox L. & Solanaceae & Terophyte & Asian & Exotic \\
\hline Nicotiana glauca Graham & Solanaceae & Nanophanerophyte & American & Exotic \\
\hline Nicotiana tabacum L. & Solanaceae & Hemicryptophyte & American & Exotic \\
\hline Solanum crispum Ruiz \& Pav. & Solanaceae & Nanophanerophyte & South American & Native \\
\hline Solanum jasminoides J. Paxton & Solanaceae & Nanophanerophyte & American & Exotic \\
\hline Solanum lycopersicum L. & Solanaceae & Terophyte & American & Exotic \\
\hline Solanum tuberosum L. & Solanaceae & Geophyte & American & Exotic \\
\hline Strelitzia reginae Banks & Strelitziaceae & Hemicryptophyte & African & Exotic \\
\hline Tamarix ramosissima Ledeb. & Tamaricaceae & Nanophanerophyte & Eurasian & Exotic \\
\hline Taxus baccata L. & Taxaceae & Phanerophyte & Eurasian & Exotic \\
\hline Tropaeolum majus L. & Tropaeolaceae & Chamaephyte & American & Exotic \\
\hline Ulmus americana L. & Ulmaceae & Phanerophyte & American & Exotic \\
\hline Zelkova carpinifolia (Pall.) K. Koch & Ulmaceae & Phanerophyte & Asian & Exotic \\
\hline Parietaria judaica L. & Urticaceae & Chamaephyte & Eurasian & Exotic \\
\hline Soleirolia soleirolii (Req.) Dandy & Urticaceae & Chamaephyte & Mediterranean & Exotic \\
\hline Urtica urens L. & Urticaceae & Terophyte & Mediterranean & Exotic \\
\hline Aloysia citriodora Palau & Verbenaceae & Nanophanerophyte & South American & Native \\
\hline $\begin{array}{l}\text { Aloysia gratissima (Gillies \& Hook.) } \\
\text { Tronc. }\end{array}$ & Verbenaceae & Nanophanerophyte & American & Exotic \\
\hline Duranta erecta $\mathrm{L}$. & Verbenaceae & Nanophanerophyte & American & Exotic \\
\hline Lantana camara $\mathrm{L}$. & Verbenaceae & Nanophanerophyte & American & Exotic \\
\hline Verbena litoralis Kunth & Verbenaceae & Terophyte & South American & Native \\
\hline Viola cornuta L. & Violaceae & Hemicryptophyte & European & Exotic \\
\hline Viola odorata L. & Violaceae & Terophyte & European & Exotic \\
\hline $\begin{array}{l}\text { Viola wittrockiana Gams ex Nauenb. \& } \\
\text { Buttler }\end{array}$ & Violaceae & Terophyte & European & Exotic \\
\hline $\begin{array}{l}\text { Parthenocissus quinquefolia (L.) } \\
\text { Planch. }\end{array}$ & Vitaceae & Phanerophyte & American & Exotic \\
\hline Vitis vinifera $\mathrm{L}$. & Vitaceae & Nanophanerophyte & Eurasian & Exotic \\
\hline Drimys winteri J.R.Forst. \& G.Forst. & Winteraceae & Phanerophyte & South American & Native \\
\hline Aloe arborescens Mill. & Xanthorrhoeaceae & Hemicryptophyte & African & Exotic \\
\hline Aloe aristata Haw. & Xanthorrhoeaceae & Hemicryptophyte & African & Exotic \\
\hline Aloe brevifolia Mill. & Xanthorrhoeaceae & Hemicryptophyte & African & Exotic \\
\hline Aloe perfoliata L. & Xanthorrhoeaceae & Hemicryptophyte & African & Exotic \\
\hline Aloe vera (L.) Burm. f. & Xanthorrhoeaceae & Hemicryptophyte & African and Asian & Exotic \\
\hline Pasithea caerulea (Ruiz \& Pav.) D.Don & Xanthorrhoeaceae & Hemicryptophyte & South American & Native \\
\hline Phormium tenax J.R.Forst. \& G.Forst. & Xanthorrhoeaceae & Hemicryptophyte & Oceanian & Exotic \\
\hline
\end{tabular}

${ }^{1}$ Nomenclature according to The Plant List (URL: http://www.theplantlist.org/ Viewed: May 4, 2015). / Nomenclatura de acuerdo a The Plant List (URL: http://www.theplantlist.org/ Viewed: May 4, 2015).

Recibido: 01.08.14

Aceptado: 10.09.15 